\title{
Fabrication, Characterization and Far-Field Optical Properties of an Ordered Array of Nanoapertures
}

\author{
Arnaud Chovin, ${ }^{\mathrm{a}}$ Patrick Garrigue, ${ }^{\mathrm{a}}$ Inka Manek-Hönninger ${ }^{\mathrm{b}}$ and Neso Sojic ${ }^{\mathrm{a},{ }^{*}}$ \\ ${ }^{a}$ Laboratoire d'Analyse Chimique par Reconnaissance Moléculaire, Université Bordeaux I, \\ ENSCPB, 16 avenue Pey-Berland, 33607 Pessac, France. E-mail : sojic@enscpb.fr \\ ${ }^{b}$ Centre Lasers Intenses et Applications, Université Bordeaux I, 351 Cours de la Libération, \\ 33405 Talence, France
}

\section{Supporting Information}

\section{General procedure for the fabrication of arrays of nanoapertures.}

The optical fiber bundle was polished before their use with 30-15-3-0.3 $\mu \mathrm{m}$ lapping films. The polished side was then placed horizontal for $5 \mathrm{~h}$ into a buffered etching solution containing $500 \mu \mathrm{l}$ of $40 \%$ (wt/wt) aqueous $\mathrm{NH}_{4} \mathrm{~F}$ solution, $100 \mu \mathrm{l}$ of a $48 \%$ HF solution and $100 \mu 1$ deionized water. (Caution: HF etching solutions are extremely corrosive!). Etched imaging fibers were rinsed and sonicated thoroughly in water to remove any residuals. The distal face of the resulting nanotip array was sputter-coated with a gold layer and connected to a copper wire with an Ag conductive paint. An identical potential is therefore applied to all the electrodes. The ensemble is then insulated on the side with a varnish. This gold-coated nanotip array is immersed in a dilute aqueous paint solution with a volume fraction 1:1 of 3 $\mathrm{mM}$ acetic acid/cathodic electrophoretic paint. The potential is scanned once from 0 to $2.5 \mathrm{~V} / \mathrm{Ag} / \mathrm{AgCl}$ at $20 \mathrm{mV} / \mathrm{s}$ between the gold-coated bundle and a $\mathrm{Cu}$ anode to reduce water at the array. The $\mathrm{pH}$ change induces the precipitation of an insulating layer on the cathode. The insulated array is cured at $180^{\circ} \mathrm{C}$ for $1 \mathrm{~h}$. While curing, the paint retracts from the tips exposing the gold electrode surface. The etching of the exposed gold layer at the tip apices is done with a common $\mathrm{KI}: \mathrm{I}_{2}$ aqueous solution for $30 \mathrm{~s}$. The nanoaperture array is eventually removed from solution and washed thoroughly in water. This procedure leads to nanoapertures of a radius $\sim 220 \mathrm{~nm}$. In order to decrease the radius, we applied two successive coatings with the electrophoretic paint and then etched the gold at the tip apex for 15 or $30 \mathrm{~s}$. Nanoapertures of $\sim 100 \mathrm{~nm}$ or $\sim 150 \mathrm{~nm}$ were thus fabricated.

\section{Epifluorescence imaging.}

The instrument used for fluorescence imaging was a modified epifluorescence microscope (BX-30, Olympus). Light from a $75 \mathrm{~W}$ Xenon arc lamp was collimated and passed through a $485 \mathrm{~nm}$ excitation filter (Omega Optical) corresponding to the Ru(bpy) ${ }_{3}{ }^{2+}$ excitation wavelength. The wavelength was then reflected at $90^{\circ}$ by a $540 \mathrm{~nm}$ dichroic mirror and focused onto the proximal end of the imaging fiber with a 20x microscope objective. Excitation light was transmitted through the optical fiber bundle and induced fluorescence emission. A fraction of the emission was collected by the optical fiber bundle, passed through the same microscope objective and was transmitted through the dichroic mirror. Fluorescence emission was filtered by a band-pass filter $(605 \mathrm{~nm})$. The fluorescence images were acquired by a back-illuminated CCD camera. 